PAPER

\title{
Phoneme Recognition Based on AF-HMMs with Optimal Parameter Set
}

\author{
Narpendyah W. Ariwardhani ${ }^{1}$, Masashi Kimura', Yurie Iribe ${ }^{1}$, Kouichi Katsurada ${ }^{1}$ and Tsuneo Nitta ${ }^{1,2}$ \\ ${ }^{1}$ Graduate School of Engineering, Toyohashi University of Technology \\ 1-1 Hibarigaoka, Tenpaku-cho, Toyohashi 441-8580, Japan \\ ${ }^{2}$ Green Computing Research Organization, Waseda University \\ 27 Waseda-machi, Shinjuku-ku, Tokyo 162-0042, Japan \\ E-mail: narpen@vox.tutkie.tut.ac.jp, iribe@imc.tut.ac.jp, katsurada@cs.tut.ac.jp,nitta@cs.tut.ac.jp
}

\begin{abstract}
We describe an improvement of the design of the phoneme recognizer that is based on the articulatory feature (AF). Several strategies for designing the optimal parameter set in AF-based Hidden Markov Model (HMM) are investigated. They include subword units, number of HMM states, vowel group separation, tuned insertion penalty, and HMM topologies. The proposed AF-based phoneme recognition with 5-state HMMs, separated vowel, triphone subword, Bakis topology, and optimal insertion penalty provides the best accuracy among the experiments, i.e., $81.38 \%$ for the JNAS speech database. This result surpasses the accuracy of the standard MFCC-based phoneme recognition for triphone subword, 3-state HMMs, and 16 Gaussian mixtures.
\end{abstract}

Keywords: speech recognition, phoneme recognition, HMM, articulatory feature

\section{Introduction}

Over the past few years, several studies have been conducted on the design of an optimal HMM configuration for automatic speech recognition (ASR). Most of these studies are based on spectral-representation feature vectors, e.g., linear predictive coding (LPC) coefficients and mel-frequency cepstrum coefficients (MFCC) [1]-[4]. On the other hand, phonetic features, such as articulatory features (AF), have proved their robustness across speakers, against co-articulatory effects, and against noise [5],[6]. Despite these advantages, the literature on the design of an optimal parameter set for AF-based HMM speech recognition is still limited.

For instance, the well-known explanation from Rabiner, which comprehensively describes HMM configurations [1], is based on LPC vectors. A more recent investigation has also yielded as an MFCC-based approach to determine acoustic model (AM) topology, i.e., the number of Gaussian mixture model (GMM) components per state and the total number of clustered states. This topic was explored in [3], where variational Bayesian estimation and clustering was implemented for large-vocabulary continuous speech recognition (LVCSR). Mitchell et al. [2] used cepstral-based vectors to investigate a variety of change functions as the cost of making a transition from one phoneme to another during Viterbi alignment.

Even though cross-language phoneme recognition performances cannot be directly compared, it is convenient to refer to the state-of-the-art phoneme recognition result to evaluate whether our experiments reach acceptable performance. In general, the accuracy of phoneme recognition still remains less than 80\% [7],[8], where the current world's best score was achieved by Mohamed et al. [9] with the accuracy of $79.3 \%$ for the TIMIT speech database.

AFs are closely linked to the physiology of a speech production mechanism. The distinctive phonetic feature (DPF), or distinctive feature, is also the most basic unit of the phonological structure, analyzed in phonological theory [10], [11], and represents the manner of articulation (e.g., vocalic, nasal, or continuant) and the tongue position (e.g., high, anterior, or back). Phonemes are viewed as a shorthand notation for a set of features that describe the behavior of the articulators required for producing distinctive aspects of a speech sound; e.g., the phonemes $/ \mathrm{p} /$ and $/ \mathrm{b} /$ are produced in ways that differ only in the state of the vocal folds. The phoneme $/ p /$ is produced without vibration (unvoiced), while $/ \mathrm{b} /$ requires the vibration of the vocal folds (voiced). In the distinctive feature representation, only the feature "voice" differs for these two sounds.

The principle of distinctive features was first proposed in the work of Jacobson et al. [11], wherein they introduced the classification scheme of the distinctive features. While Espy-Wilson and Bitar [12] measured the properties of the signal, such as energy, in certain frequency bands and formant frequencies, and defined the 


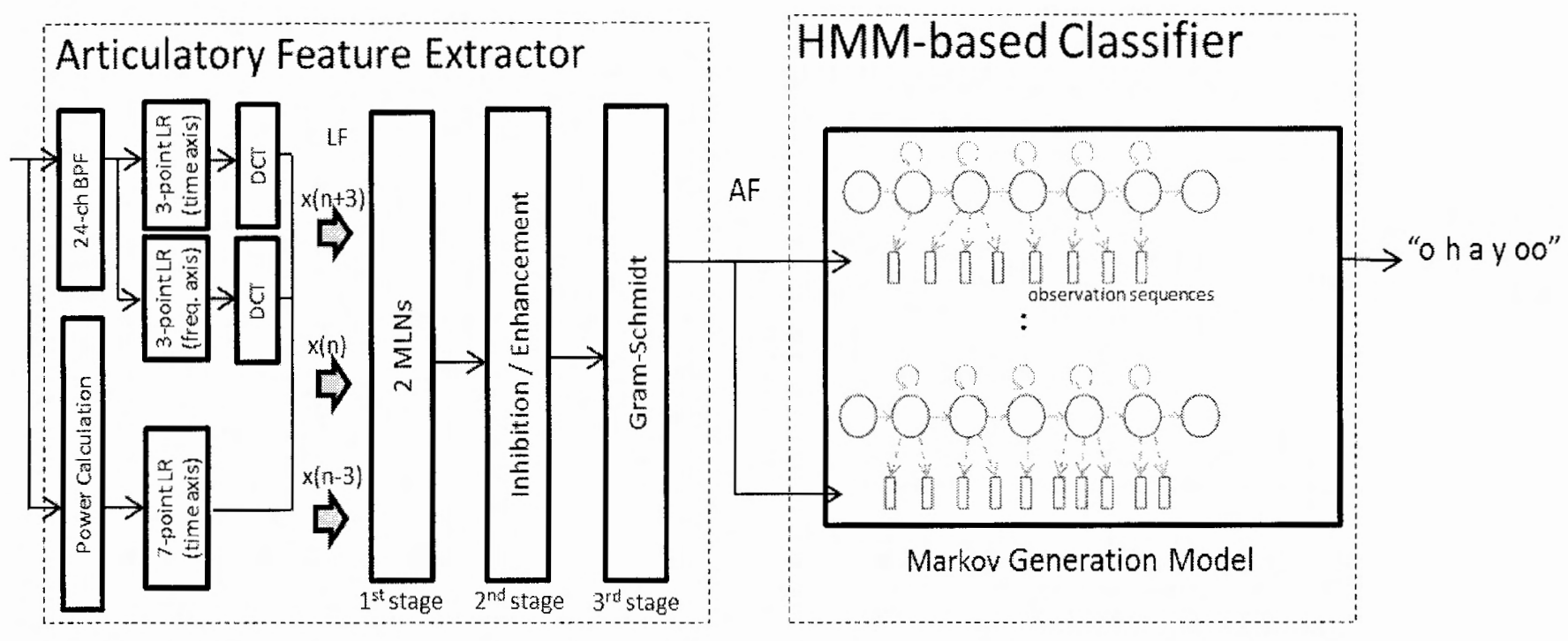

Fig. 1 Phoneme recognition engine

phonetic features as functions of these acoustic measurements. Kirchhoff et al. [13] proposed a system in which a neural network is used to predict manner and place features. The work showed that the feature-based recognizer performed comparatively better under noisy conditions and that a combination of a phone-based recognizer and feature recognizer was better than either alone. Eide [14] described, in his work, that combining the distinctive feature representation with the standard cepstral representation improved automatic speech recognition performance.

A typical phoneme recognition system can be divided into two parts, i.e., a feature extractor and an HMM-based classifier. Subsequent to our previous works of a DPF extractor, or an AF extractor [15], [16], this paper will describe further our experimental studies on the design of an optimal HMM-based classifier. The aim of this work is to establish the design of AF-based HMMs through a comparative investigation of AF-based phoneme recognition and the MFCC-based approach.

In this work, we focus on phoneme recognition rather than word recognition to develop ASR systems that can adapt to out-of-vocabulary (OOV) words in the near future. Our goal is to improve the phoneme accuracy performance, by investigating the optimal parameters that affect the AF-based phoneme recognition, i.e., subword units, number of HMM states, vowel group separation, tuned insertion penalty, and HMM topologies.

This paper is organized as follows. First, an outline of speech recognition based on AF-HMMs is reviewed in Section 2. Our strategies for improving AF-based HMM phoneme recognition is described in Section 3. Next, an evaluation of phoneme recognition, in which we show and

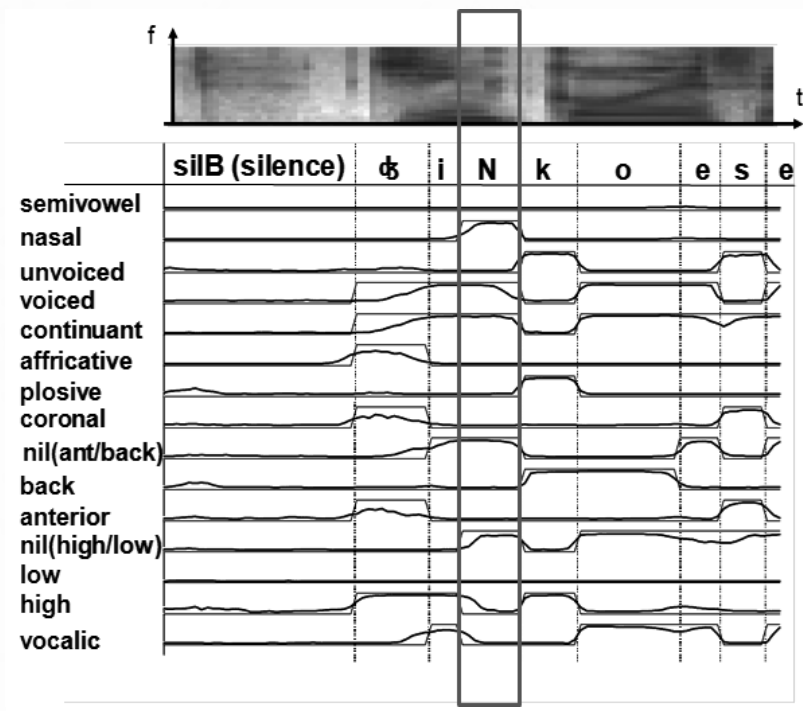

Fig. 2 Articulatory feature sequence /jiNkooeiseil (artificial satellite)

discuss experimental results for each approach, is given in Section 4. Finally, we summarize our finding in Section 5.

\section{Speech Recognition Based on AF-HMMs}

The proposed speech recognition engine is divided into two parts: a three-stage AF extractor, which converts input speech into AFs [16], and an AF-based HMM classifier (Fig. 1). The first stage of the AF extractor extracts 45-dimensional AF vectors from the local features (LFs) of the speech input using two multilayer neural networks (MLNs). The second stage incorporates 
the inhibition/ enhancement functionalities, and the third stage decorrelates three context vectors by Gram-Scmidht (GS) orthogonalization before connecting with the HMMbased classifier [16]. LFs represent variation in a spectrum pattern along two axes: time axis and frequency axis [17]. We use LFs for the input of MLN, as our previous study showed that LFs provides better performance than MFCC as input to this MLN [18].

There are two MLNs used to convert LFs to AFs. The first MLN (MLN $\left.{ }_{L F-A F}\right)$ is to map LFs onto AFs, while the second MLN (MLN $\mathrm{N}_{\text {Dyn }}$ ) is to reduce misclassification at the phoneme boundaries by constraining AF context. The input for $\mathrm{MLN}_{\mathrm{LF}-\mathrm{AF}}$ is LFS vectors, whereas the input for $M \mathrm{~N}_{\text {Dyn }}$ is 45-dimensional context-dependent AF vectors provided by the precedent $M L N_{L F-A F}$ output and appended with its corresponding $\triangle \mathrm{AF}$ and $\triangle \triangle \mathrm{AF}$ vectors. The latter dynamic AF vectors are calculated using 3-point linear regression.

Both MLNs comprise four layers, including two hidden layers. These MLNs are trained using the backpropagation algorithm with AF vectors (derived from label data) as their correct target. Fig. 2 shows an example of the AF sequence for the utterance /jiNkooeisei (artifical satellite)/. In the figure, 15 elements of Japanese AFs are shown. For instance, phoneme $/ \mathrm{N} /$ is described as nasal, voiced, and continuant. A "solid thin line" represents ideal segmentation, whereas a "solid bold line" represents the extracted AF sequences at the first stage of the AF extractor.

The output of the AF extractor still contains temporal variability, which is handled by the second part of our speech recognition. For this issue, we use the conventional HMM approach. On the HMM-classifier side, some information is needed to define a single HMM, i.e., the type of observation vector, number of states, and transition matrix. In the baseline experiment, we use a simple left-to-right HMM with three emitting states (five states in total, including an entry state and an exit state with no self-loop), so that the transition matrix for this model has five rows and five columns.

Flat-start initialisation is used, in which the global mean and variance are assigned to every Gaussian distribution in every phoneme HMM. This implies that during the first cycle of the embedded re-estimation, each training utterance will be uniformly segmented. Subsequently, the Baum-Welch training process is adopted to estimate the parameters of the HMMs from examples of the data sequences that correspond to the models. We use embedded training in which the training simultaneously re-estimates the occupation probability in a complete set of subword HMMs. For each input utterance, all the subword HMMs corresponding to the phone list in that utterance are joined to make a single composite HMM. This composite HMM is used to collect the necessary statistics for the re-estimation.
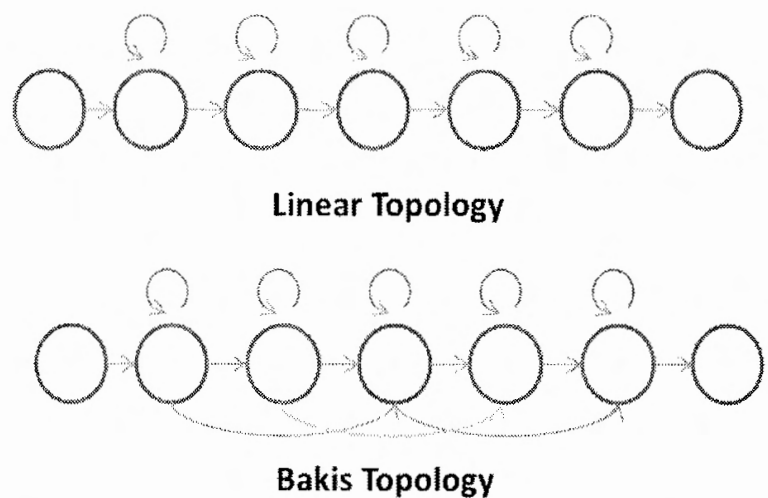

Fig. 3 Schematic representation of linear and Bakis HMM topologies

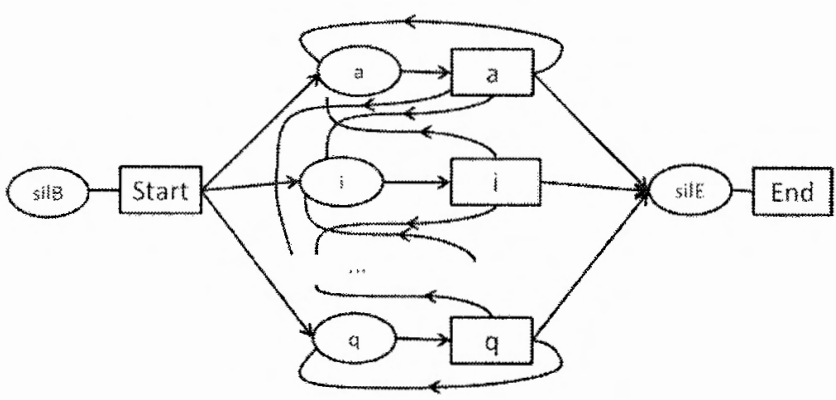

(a)

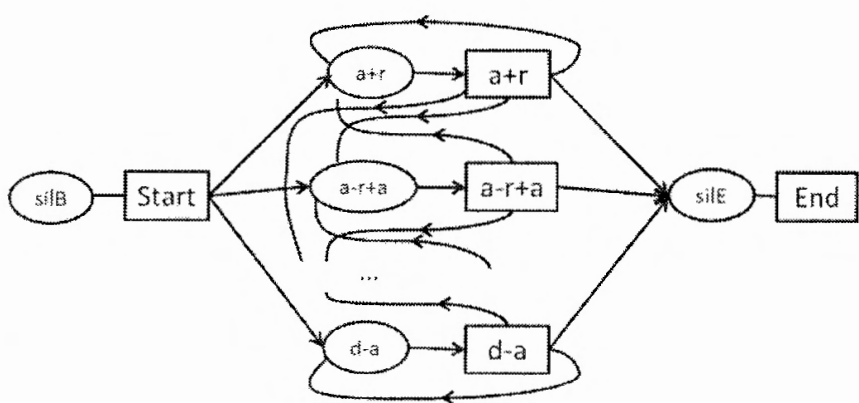

(b)

Fig. 4 Recognition network of (a) monophone and (b) word internal triphone

For model refinement, we use a typical approach, i.e., the conversion of a set of initialised and trained contextindependent monophone HMMs to a set of contextdependent models. We conducted triphone construction, which involved cloning all monophones and then reestimating them using the data for which monophone labels have been replaced by triphone labels. We built a set of word internal context-dependent (triphone) models in which the word boundaries in the training transcriptions are marked. 
Given a recognition network, its associated set of HMMs, and unknown utterances, we can calculate the probability of any path through the network. The task of a decoder (Viterbi) is to find the most likely paths. In the end, we evaluate the performance of the phoneme recognizer using a test database and a set of reference transcriptions to compute the correct rate and the accuracy of phoneme recognition.

\section{Improving Phoneme Recognition Performance}

The task of phoneme recognition is to convert speech to a phoneme string rather than words. While ASR relies heavily on contextual constraints (i.e., language model (LM)) to guide the search algorithm, the phoneme recognition task is much less constrained than word decoding, and therefore, the error rate (even when measured in terms of the phoneme errors for word decoding) is considerably high. To improve the phoneme recognition performance, we conduct several approaches as described below.

\subsection{Optimal state configuration and parameter set}

Besides extending monophone HMMs to triphone HMMs, we extend 3-state (3-loop) HMMs to 5-state (5loop) HMMs and evaluate their performance. On the other hand, Japanese vowels are of two types according to their duration, namely, short vowels and long vowels. To deal with the difference in the standard deviations, we separated the short vowels and the long vowels using labeled data. A vowel that has larger pronunciation duration than its class average value will be relabeled as a long vowel, and vice versa. These separated vowels will be treated as different phonemes during the HMM training and the beginning of the testing phase. However, these separated vowels will be re-unified after the recognition phase.

In the experiments, two HMM topologies were used, i.e., a linear HMM topology and the Bakis topology (Fig. 3). In the linear HMM topology, only the transitions to the next state and the current state are possible with some positive probability. Using the self-transitions or loops, the model is able to capture variations in the temporal extension of the patterns described.

The linear HMM topology is the simplest model. Linear models represent the most efficient model topology, as for the other models, not only does the parameter training become more difficult for a large number of successors per model state but the effort needed in decoding is also increased [19]. Meanwhile, the Bakis topology allows larger flexibility in the modeling of the duration by making it possible to skip the individual states.

\subsection{Insertion penalty in decoding process}

The HTK toolkit [20] is used to construct the acoustic model. In the toolkit, decoding is controlled by a recognition network compiled from a word-level network, a dictionary, and a set of HMMs. To build a phoneme recognizer, a word-level network is defined in the usual manner, except that each "word" in the network represents a single phoneme. The structure of the network will typically be a loop in which all phonemes loop back to each other (Fig. 4). In the figure, the oval frames denote the HMM instances and the square frames denote the phoneme-end nodes. In this network, the Token Passing algorithm [21] is applied to find the best path and generate the hypothesis. In every HMM state, the tokens are examined, and only the token with the highest probability is preserved.

As a token is propagated from the exit state of a phoneme to the entry state of another, this transition represents a potential phoneme boundary. At this point, a fixed (insertion) penalty $\beta$ is added to the tokens emitted from the corresponding phoneme-end node.

$$
\log p(\boldsymbol{O} \mid w)+\beta
$$

For example, adding an insertion penalty of -30 means adding a value of -30 to the tokens emitted from the corresponding phoneme-end node.

Within HMM, transitions are determined from the HMM parameters, whereas for the phoneme-end, transitions are controlled by scaling the language model likelihoods and adding a fixed penalty. Since we focus on the acoustic model, we do not implement the language model. Therefore, adding a fixed penalty would require a more significant value than the typical one. This fixed penalty will control the relative levels of the insertion and deletion errors of the phoneme recognizer.

\section{Evaluation of Phoneme Recognition}

\subsection{Speech database}

The following three clean speech datasets are used in our experiments.

\section{(1) Dl dataset for training MLN}

This dataset contains 4503 sentences from subset A of the Acoustic Society of Japan (ASJ) Continuous Speech Database [22]; the sentences were uttered by 30 male speakers $(16 \mathrm{kHz}, 16 \mathrm{bit})$.

\section{(2) D2 dataset for training HMM}

This dataset contains 5000 sentences that have been taken from the Japanese Newspaper Article Sentences (JNAS) [23] Continuous Speech Database; the sentences have been uttered by 33 different male speakers $(16 \mathrm{kHz}$, 16 bit).

(3) D3 dataset for testing HMM

This test dataset comprises 2379 JNAS sentences uttered by 16 male speakers ( $16 \mathrm{kHz}, 16$ bit). Speakers in the D3 dataset are different from those in the D2 dataset. 


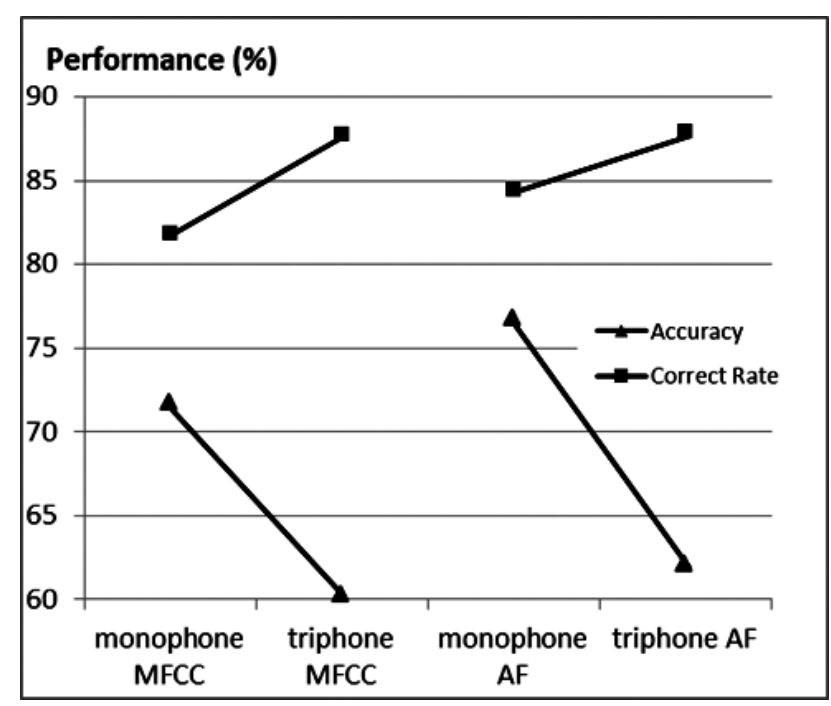

Fig. 5 Extending subword unit from monophone to triphone on 3-state HMMs (16 mixtures)

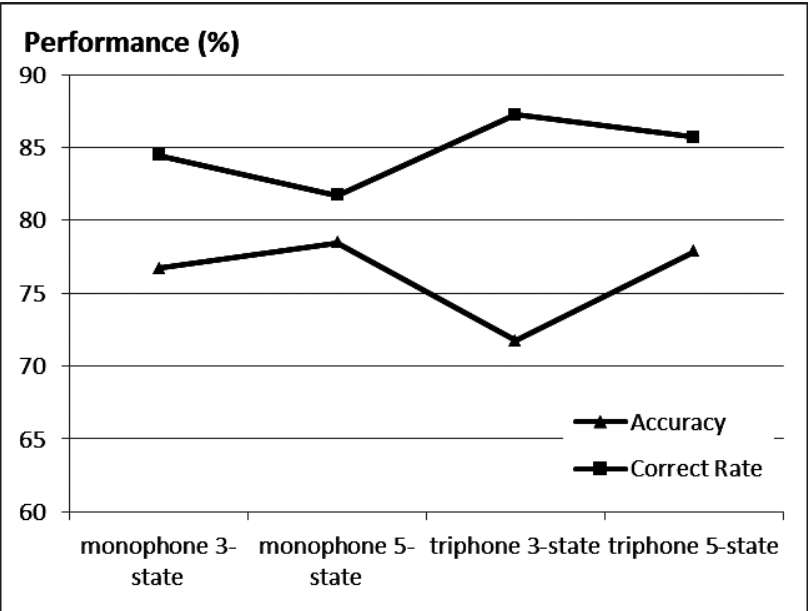

Fig. 6 AF-based accuracy improvement from 3-state HMMs to 5-state HMMs (16 mixtures)

\subsection{Experimental setup}

The frame length and the frame rate were set to $25 \mathrm{~ms}$ and $10 \mathrm{~ms}$, respectively. The phoneme recognizer performance was measured by its correct rate and accuracy. The correct rate is the percentage of correct labels out of the total number of labels, without taking insertion errors into consideration. Its formulation is defined as

$$
\text { Correct Rate }=\frac{N-D-S}{N} \times 100 \%
$$

where $\mathrm{N}$ is the total number of labels in the reference transcriptions, $\mathrm{D}$ is deletion error, and $\mathrm{S}$ is substitution error. On the other hand, accuracy is considered as a more representative figure of recognizer performance, because it also takes insertion errors (I) into account, as defined below.

$$
\text { Accuracy }=\frac{N-D-S-1}{N} \times 100 \%
$$

For vowel-unified Japanese monophones, the D2 dataset was used to design $38 \mathrm{HMMs}$, whereas for the separated Japanese monophones, the D2 dataset was used to design 43 Japanese monophones. Unified vowels were reused during the recognition.

In the HMMs, the output probabilities were represented in the form of Gaussian mixtures, and diagonal matrices were used. The number of mixture components in the HMM was varied among $1,2,4,8$, and 16 .

Two different feature vectors were used to evaluate this phoneme recognition system. The first type of feature vector used is the standard MFCC-feature set, which consists of a vector with 39 dimensions $(12 \mathrm{MFCC}+\log$ energy of the speech signal, $\Delta$ and $\Delta \Delta$ coefficients). The second one is the AF-vector with 45 dimensions (15 preceding-context AF patterns, 15 current-frame AF patterns, and 15 following-context AF patterns) for each input frame.

\subsection{Experimental results and discussion}

The typical motivation of extending monophone to triphone comes from the classical idea of coarticulation, i.e., the concept that speech sound is influenced by its preceding or following speech sound. Even though during the extraction of AF we incorporated three contextdependent frames (in order to solve the coarticulation problem), in the experiment, we still found an improvement in the correct rate when extending monophone to triphone. However, this improvement was not followed by an accompanying improvement of accuracy. The accuracy of phoneme recognition decreased significantly (Fig. 5). This accuracy degradation while extending monophone to triphone indicates that a large insertion error occurred. Insertion errors occur when the system recognizes phonemes that do not occur. These errors are different from the deletion errors, which arise when the system fails to recognize the occurrence of phonemes within a stream of data. A better performance of phoneme recognition can be obtained by balancing the deletion errors and the insertion errors. To balance these two errors, the insertion penalty value can be tuned into its optimal value. These tuned penalty results will be discussed later.

The number of states in the HMM configuration is a matter of choice. A low number of states makes it easier to learn the model but may cause underfitting, whereas too many states make it harder to learn and may overfit the noise. In the experiment, we compare the performance of 3-state HMMs to 5-state HMMs. As can be seen from Fig. 6, extending 3-state HMMs to 5-state HMMs decrea- 


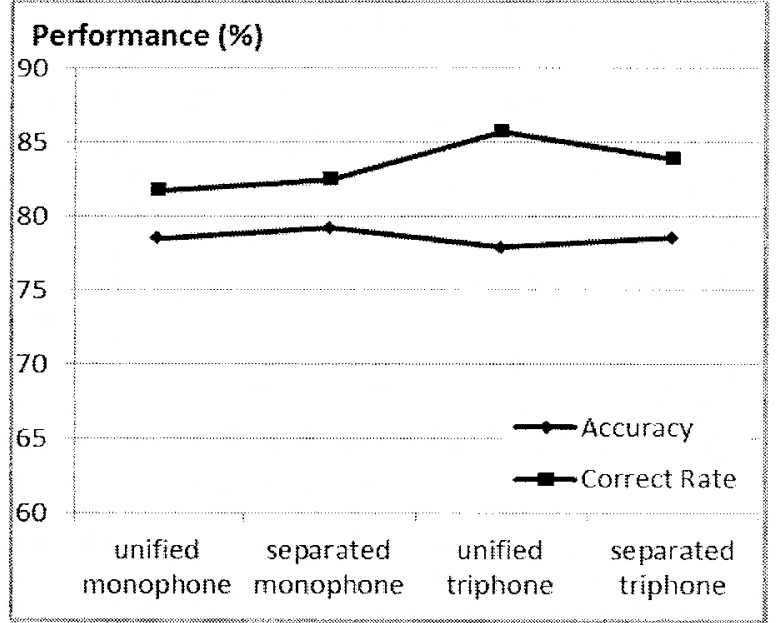

Fig. 7 AF-based 5-state (16 mixtures) HMM phoneme recognition accuracy improvement from unified vowels to separated vowels
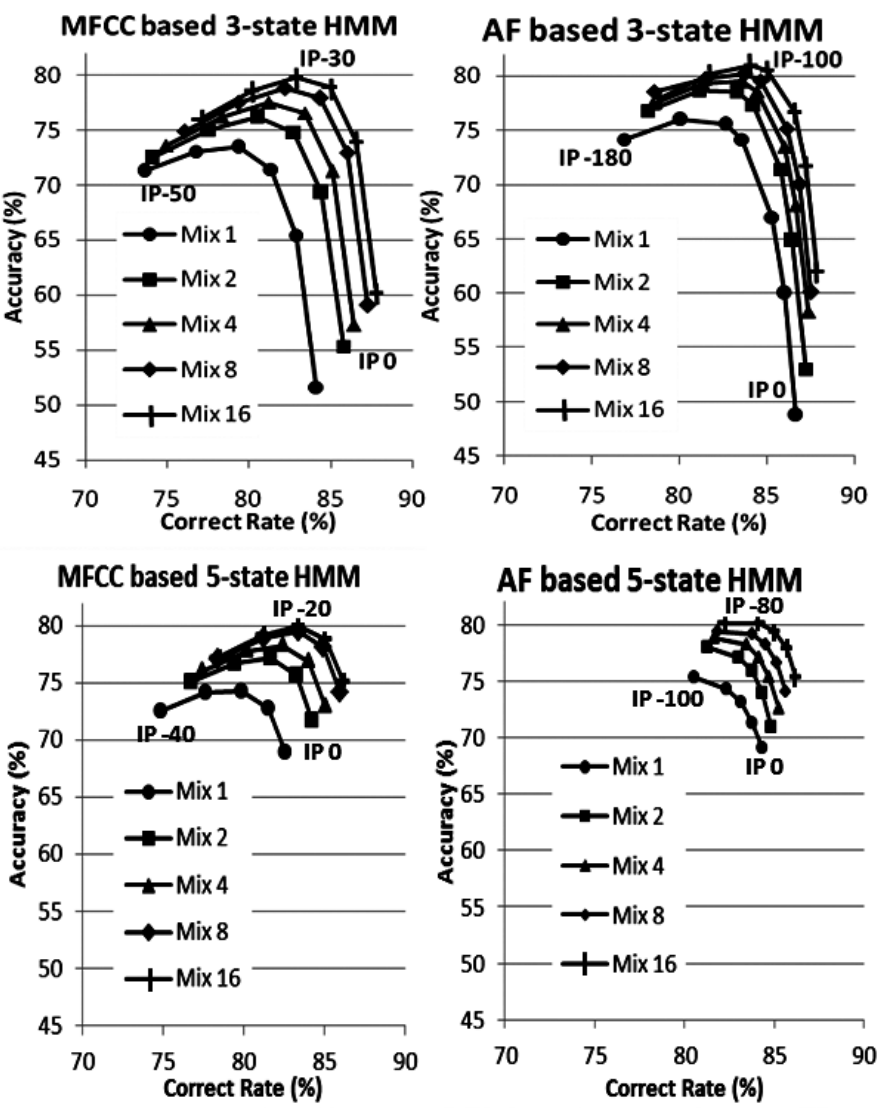

Fig. 8 Phoneme recognition accuracy vs correct rate at different insertion penalty values on triphone HMM unified vowels

sed the correct rate performance, yet increased the accuracy. Since accuracy measurement takes insertion error into account, it describes the phoneme recognition

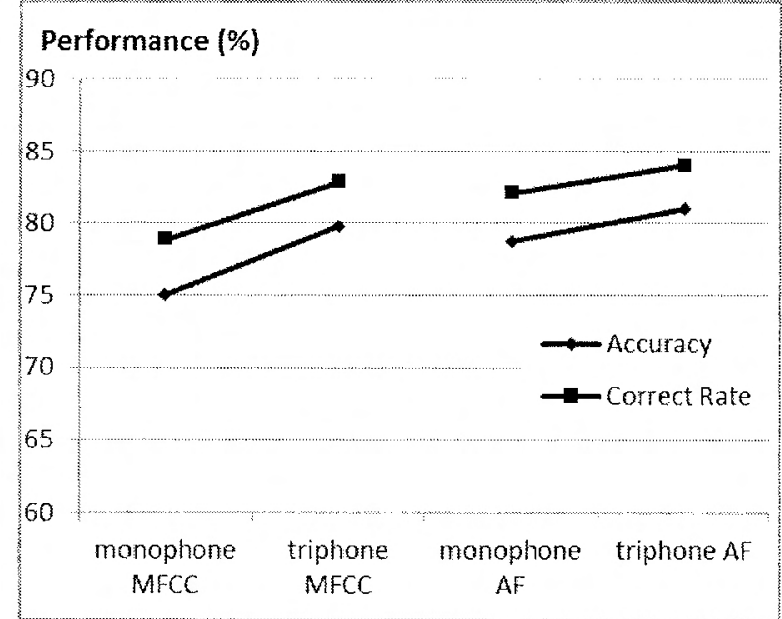

Fig. 9 3-state (16 mixtures) HMM phoneme recognition performance improvement by tuning optimal insertion penalty

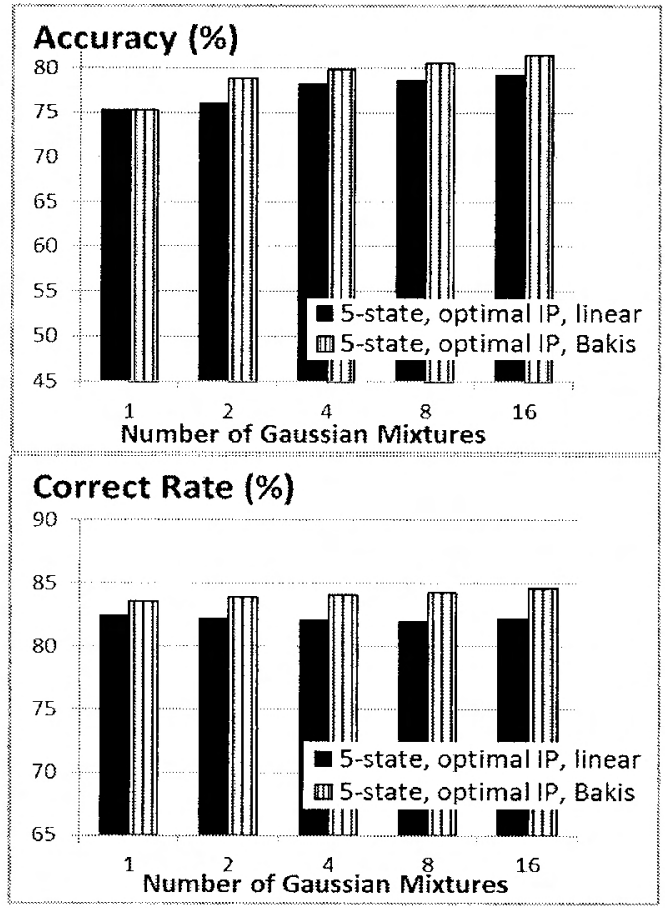

Fig. 10 AF-based phoneme recognition performance from linear topology to Bakis topology

performance more comprehensively than does the correct rate. We focus on improving the accuracy of the phoneme recognition; thus, the next approach will follow 5-state HMMs.

Figure 7 shows the accuracy for AF-based 5-state HMM phoneme recognition. By separating vowels into short vowels and long vowels, we can improve the accuracy of phoneme recognition. On the monophone side, this vowel separation technique also improves the 


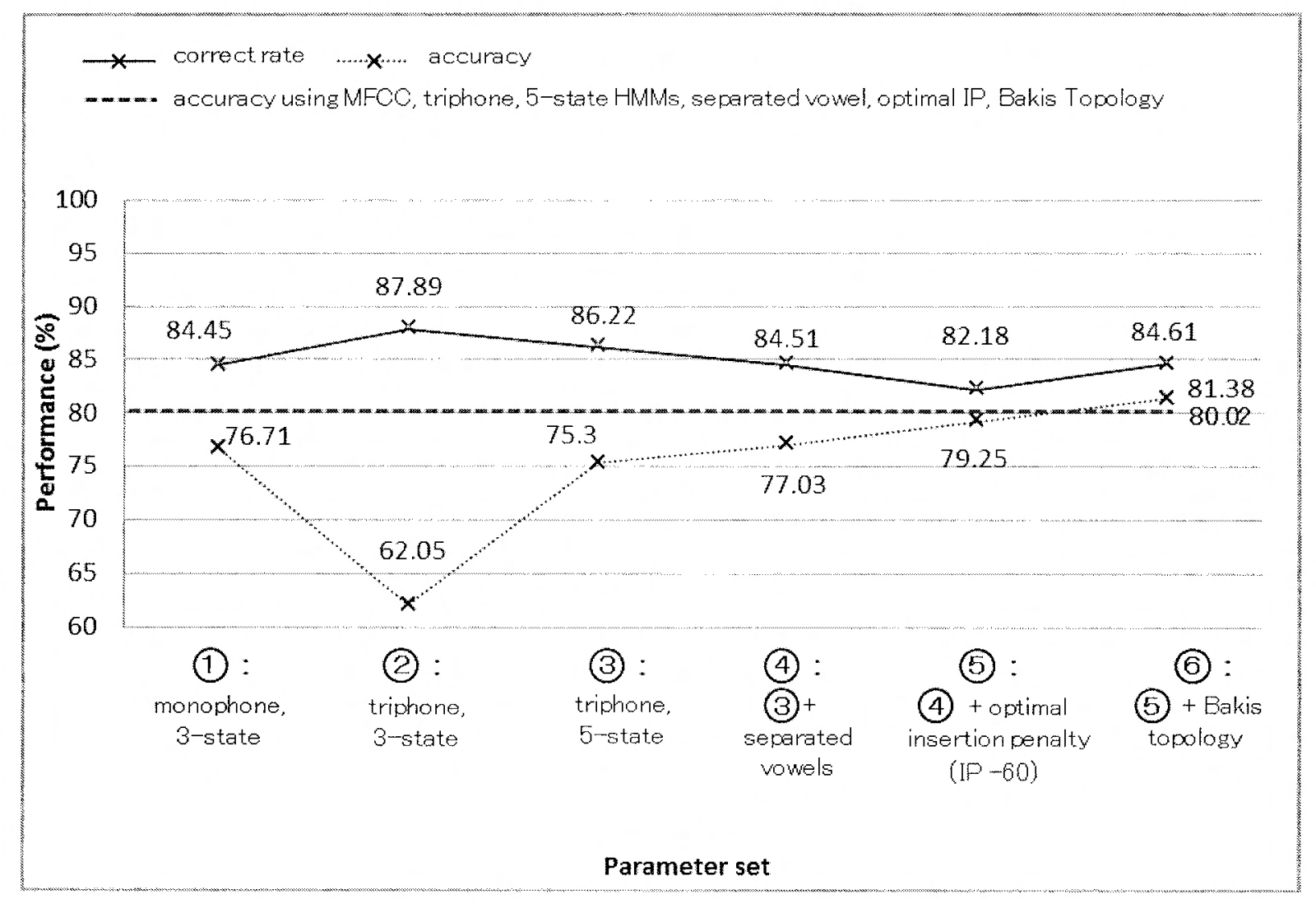

Fig. 11 Performance progress of AF-based HMM phoneme recognizer on 16 components of Gaussian mixtures for different parameter sets

correct rate of phoneme recognition. The insertion penalty is a tuning parameter to control the transition from the end state of one phoneme to the start states of all the other phonemes. The insertion penalty penalizes insertions that occur between phonemes. We gave large negative values of the insertion penalty, which lowers the probability of a phoneme so that a large number of phonemes are not hypothesized randomly. This may decrease insertion errors but may increase the deletion errors.

Figure 8 shows the advantage of AF compared with MFCC. This figure shows that we can improve the accuracy of the AF-based phone recognition by tuning the insertion penalty without significantly decreasing its correct rate. On the MFCC side, this tuning reduces the correct rate significantly. Moreover, in this figure, compared with the 3-state HMM phoneme recognizer, the 5-state HMM phoneme recognizer is shown to be less sensitive to insertion error as it is more unlikely to recognize additional longer sequences of HMM. This also occurs in a typical HMM speech recognizer, where the speech recognizer tends to favor shorter words during insertion error.

The larger IP value occurring in AF means that the transition from the end state of one phoneme to the start states of all the other phonemes is more likely to happen. This may be because $A F$ is a discrete-like vector where the difference between AF vectors of one phoneme and other phonemes might occur only in a few, one, or two, components of AF. Therefore, when the averages of observation vectors $(A F)$ are similar in these boundary states, the transitions are more likely to occur. To overcome this problem, the larger IP value is needed to penalize these transitions. As a result of insertion penalty tuning, Fig. 9 shows the phoneme recognition performance (for both accuracy and correct rate) improvement obtained by extending a monophone to a triphone.

Previous experiments were conducted on the linear topology of HMM. After determining that separating vowels and increasing HMM states to 5-states result in an accuracy improvement, we also investigated the influence of the HMM topology on the phoneme recognizer performance. Figure 10 shows that, compared with the linear topology, the Bakis topology worked well for improving both the correct rate and the accuracy of the AF-based phoneme recognition. These improvements result from the flexibility of Bakis topology, i.e., the possibility of skipping the individual states. This 
flexibility allows us to model duration, particularly when phonemes do not have similar durations. This Bakis length modeling method optimizes the predefined number of HMM states. We have conducted some combinations from the parameters explained. The performance improvement of the AF-based HMM phoneme recognizer for 16 components of Gaussian mixtures is described in Fig. 11.

It is widely known that in order to have good speech recognition performance, we must balance the acoustic (insertion penalty) and linguistic (language weight) parameters. The insertion penalty (IP) in a phoneme model controls the transition from the final state to the initial state of the following phoneme model. Because in the 3-state triphone model based on AF, the difference between averaged vectors in the final state and in the succeeding state is very small, the accuracy of the 3-state triphone model before tuning the IP value is very low and is improved largely after tuning $(\mathrm{IP}=100)$. The same control is also realized by adding states (from 3-state HMM to 5-state HMM); a more detail effect of adding states has been described in Fig. 8. Furthermore, as phoneme recognition in this paper does not use a language model, the insertion penalty plays a significant role. Ignoring this penalty causes worse accuracy, as shown by the second parameter set of Fig. 11.

The best accuracy rate $(81.38 \%)$ among our experiments was attained by combining the parameters explained above, i.e., AF-based phoneme recognition for a 5-state triphone with separated vowels, optimal insertion penalty, and Bakis HMM topology. This rate is an improvement from our previous work accuracy of the AFbased phoneme recognition for a 3-state monophone with the default insertion penalty $(76.71 \%)$. For comparison purposes, we provide a line showing the accuracy value of our phoneme recognition experiment the using standard feature vectors, MFCC. The experiment was held for a triphone subword, separated vowels, 5-state HMMs, optimal insertion penalty, Bakis Topology, and 16 Gaussian mixtures $(80.02 \%)$.

\section{Conclusions}

By extending the sub-word unit monophone HMMs to triphone HMMs, the number of HMM states, conducting vowel separation (of short vowels and long vowels), tuning the insertion penalty, and applying the Bakis HMM topology, we could improve the AF-based phoneme recognition accuracy. Future works will be further improvement in the articulatory feature extractor and the HMM classifier with other probability distribution types for the AF-based approach.

\section{Acknowledgments}

This work was supported in part by the Global COE program "Frontiers of Intelligent Sensing," MEXT, Japan.

\section{References}

[1] L. R. Rabiner: A tutorial on hidden markov models and selected applications in speech recognition, Proc. IEEE, Vol. 77, No. 2, pp. 257-286, 1989.

[2] C. D. Mitchell, M. P. Harper and L. H. Jamieson: Using explicit segmentation to improve HMM phone recognition, Proc. ICASSP'95, pp. 229-232, 1995.

[3] S. Watanabe, A. Sako and A. Nakamura: Automatic determination of acoustic model topology using variational bayesian estimation and clustering for large vocabulary continuous speech recognition, IEEE Trans. Audio, Speech, and Language Processing, Vol. 14, No. 3, pp. 855-872, 2006.

[4] P. Bhuriyakorn, P. Punyabukkana and A. Suchato: A genetic algorithm-aided hidden markov model topology estimation for phoneme recognition of thai continuous speech, Proc. $9^{\text {th }}$ ACIS International Intelligence, Networking, and Parallel/Distributed Computing, pp. 475-480, 2008

[5] N. N. Bitar and C. Y. Espy-Wilson: Knowledgebased parameters for HMM speech recognition, Proc. ICASSP, Atlanta, GA, pp. 29-32, 1996.

[6] K. Kirchhoff: Robust speech recognition using articulatory information, Ph.D. Dissertation, Univ. of Bielefeld, Bielefeld, Germany, 1999.

[7] M. R. A. Kotwal, F. Hassan, G. Muhammad and M N. Huda: Tandem MLNs based phonetic feature extraction for phoneme recognition, International Journal of Computer Information Systems and Industrial Management Applications, Vol. 3, pp. 088-095, 2011.

[8] S. M. Siniscalchi, D. C. Lyu and C. H. Lee: Experiments on cross-language attribute detection and phone recognition with minimal target-specific training data, IEEE Trans. Audio, Speech, and Language Processing, Vol. 20, No. 3, pp. 875-887, 2012 .

[9] A. Mohamed, G. Dahl and G. Hinton: Acoustic modeling using deep belief networks, IEEE Trans. Audio, Speech, and Language Processing, Vol. 20, pp. 14-22, 2012.

[10] R. Jakobson, G. M. C. Fant and M. Halle: Preliminaries to Speech Analysis: The Distinctive Features and Their Correlates, MIT Press, 1952.

[11] N. Chomsky and M. Halle: The Sound Pattern of English, New York, Harper and Row, 1968.

[12] C. Y. Epsy-Wilson and N. N. Bitar: Speech parameterization based on phonetic features: application to speech recognition, Proc. Eurospeech-95, Madrid, pp. 1411-1414, 1995.

[13] K. Kirchhoff: Combining acoustic and articulatory feature information for robust speech recognition, Speech Communication, Vol. 37, pp. 303-319, 
2002.

[14] E. Eide: Distinctive features for use in an automatic speech recognition system, Proc. Eurospeech 2001, Vol. III, pp. 1613-1616, 2001.

[15] M. N. Huda, K. Katsurada and T. Nitta: Phoneme recognition based on hybrid neural networks with inhibition/enhancement of distinctive phonetic feature (DPF) trajectories, Proc. Interspeech '08, pp. 1529-1532, 2008.

[16] M. N. Huda, H. Kawashima and T. Nitta: Distinctive phonetic feature (DPF) extraction based on MLNs and inhibition/enhancement network, IEICE Trans. Inf. \& Syst., Vol. E-92D, No. 4, pp. 671-680, 2009.

[17] T. Nitta: Feature extraction for speech recognition based on orthogonal acoustic-feature planes and LDA, Proc. ICASSP, pp. 421-424, 1999.

[18] T. Fukuda, W. Yamamoto and T. Nitta: Distinctive phonetic feature extraction for robust speech recognition, Proc. ICASSP vol II, pp. 25-28, 2003.

[19] G. A. Fink: Markov Models for Pattern Recognition: from Theory to Applications, Springer, pp. 127-128, 2008.

[20] HTK Speech Recognition Toolkit (version 3.4) http://htk.eng.cam.ac.uk/

[21] S. J. Young, N. H. Russell and J. H. S. Russel: Token passing: a simple conceptual model for connected speech recognition systems, Tech. Report, Cambridge Univ. Eng. Dept., 1989.

[22] T. Kobayashi, S. Itahashi, S. Hayamizu and T. Takezawa: ASJ continuous speech corpus for research, Acoustic Society of Japan Trans. Vol, 48, No. 12, pp. 888-893, 1992.

[23] JNAS: Japanese Newspaper Article Sentences. http://www.mibel.cs.tsukuba.ac.jp/ 090624/jnas/in struct.html.

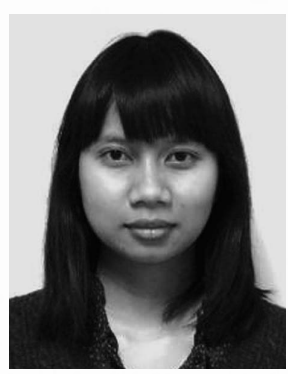

Narpendyah Wisjnu Ariwardhani received her $B$. Eng. degree and $M$. Eng. degree from Bandung Institute of Technology, Bandung, Indonesia. She is currently a doctoral student at the Graduate School of Engineering, Information and Electronic Engineering, Toyohashi University of Technology, since 2010. Her research interest includes speech recognition. She is a member of ASJ.

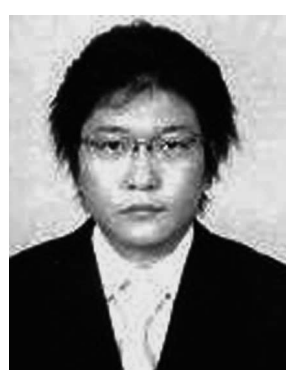

Masashi Kimura received his B. Eng. degree and M. Eng. degree from Toyohashi University of Technology, Japan. He has finished his coursework and is in the final year of his doctoral study, in-waiting for the oral examination from Graduate School of Engineering, Information and Electronic Engi- neering, Toyohashi University of Technology. Currently he is also a researcher for industry-academia-government collaboration at Toyohashi University of Technology, Japan. His research interests include speech synthesis, speech recognition and intelligent agent. He is a member of JSAI, IEICE, ASJ and IPSJ.

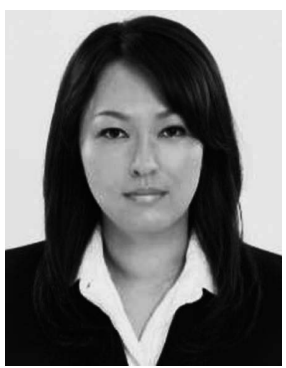

Yurie Iribe received her M.S. and $\mathrm{Ph} . \mathrm{D}$. degrees from the Graduate School of Human Informatics of Nagoya University, Japan. She is currently an Assistant Professor at the Information and Media Center, Toyohashi University of Technology, Japan. Her recent research interests include education support and speech recognition. She is a member of ISCA, IEICE, IPSJ, JSAI, and ASJ.

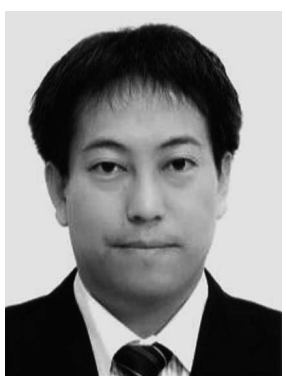

Kouichi Katsurada received his Ph.D. degree from Osaka University in 2000. He has been with Toyohashi University of Technology since 2000. He is currently an Associate Professor at the Center for International Relations and Department of Computer Science and Engi-neering, Toyohashi University of Technology. His research interests include multimodal interaction, facial image processing, and spoken term detection. He is a member of IEEE, ISCA, IEICE, IPSJ, JSAI, and ASJ.

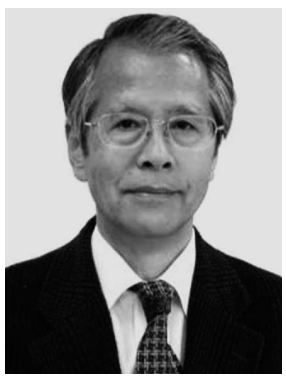

Tsuneo Nitta received his Ph.D. from Tohoku University, Japan. He had worked at R\&D Center and Multimedia Eng. Lab. of Toshiba Corp. from 1970 to 1998 . He subsequently joined Toyohashi University of Tech-nology as a Professor in the Graduate School of Eng. In 2012, he became a TUT Professor Emeritus and a visiting professor both at TUT and Waseda University. His current research interest includes speech recognition, speech synthesis, and multi-modal interaction. He is an IPSJ Fellow and a member of IEICE, ASJ, JSAI, and IEEE.

(Received May 14, 2012; revised August 10, 2012) 